\title{
Impressum, Vol. 10, No. 5, 1987
}

\section{s karger}

Verlag für Medizin und Naturwissenschaften GmbH, Postfach 1724, D-8034 Germering

Presserechtlich verantwortlich: Walter Kunz, Gesellschafter

Wissenschaftlicher Beirat

H. W. Bauer, Berlin: W. Bossnev, Sofia/Bulgarien; H. Denck, Wien; V. Diehl. Köln; P. Drings. Heidelberg; E. Dühmke, Göttin-gen: S. Eckhardt, Budapest; H. Ehrhart, München; H. H. Fiebig, Freiburg; W. M. Gallmeier, Nürnberg; E. H. Graul, Marburg/ Lahn; R. Hartenstein. München; H. Heimpel, Ulm; K. P. Hellrie-gel. Berlin; Ch. Herfarth, Heidelberg; H, W. von Heyden, Ein-beck; D. Hoelzer, Frankfurt/M.; J. H. Holzner, Wien; R. Hünig. Basel; W. Hunstein, Heidelberg; H. J. Illiger, Oldenburg; U. R. Kleeberg, Hamburg: H. O. Klein, Köln; B. Kornhuber, Frankfurt/M.: H. Löffler, Kiel: U. Mohr, Hannover; K. Munk, Heidelberg; G. A. Nagel, Göttingen; J. P. Obrecht, Basel; A. Pfleiderer, Freiburg: K. Possinger, München; W. Queißer, Mannheim; H. Riehm, Hannover; E. Scherer, Essen; M. Schroder, Göttingen; S. Seeber, Leverkusen; H. J. Senn, St. Gallen: A. Stacher, Wien; V. Sturm. Heidelberg; St. Tanneberger, Berlin/DDR: W. Vahlensieck, Bonn; H. D. Waller, Tubingen: M. Wannenmacher, Freiburg: W. Wilmanns, München; K. Wilms, Würzburg; K. zum Winkel, Heidelberg; H. Wrba, Wien.

Schriftleitung: S. Eckhardt, Budapest J.H. Holzner, Wien G. A. Nagel, Göttingen

Die Zeitschrift erscheint zweimonatlich; pro Jahr erscheint 1 Band zu je 6 Heften.

Bezugspreis für Jahrgang 10, 1987, DM 148,-/öS 1036,-/SFr 116,-. 1 Einzelheft kostet DM 28,/öS 196,-/SFr 22,-, einschließlich MwSt., zuzüglich Postgebühren.

Der Abonnementspreis ist im voraus zahlbar. Das Abonncment der Zeitschrift läuft weiter, wenn sie nicht spätestens 4 Wochen vor Abschluß eines Bandes abbestellt vvird.

Abonnementsbestellungen können bei jeder Buchhandlung, bei der Post oder direkt beim Verlag aufgegeben werden: Bundesrepublik DeutschlandlÖsterreich: S. Karger GmbH, Postfach 1724, D-8034 Germering/München, Tel.: (089) 844021. Telex: 524865 D. Postschcckkonto: München 40080-807 Schweiz: S. Karger AG. Allschwilerstr. 10, Postfach. CH-4009 Basel, Tel.: (061) 390880, Telex $62652 \mathrm{CH}$

Anzeigen

S. Karger Verlag für Medizin und Naturwissenschaften GmbH, Postfach 1724, D-8034 Germering, Telefon (089) 844021. Gültig ist die Preisliste Nr. 5 vom 1.10.1986.

Für den Inhalt außerhalb des redaktionellen Tciles (insbesondere Anzeigen.

Industrieinformationen, Pressezitate und Kongreß-informationen) übernehmen Schriftleitung. Beirat und Verlag keine Gcwähr.

Eine Markenbezeichnung kann warenzeichenrechtlich geschützt sein. auch wenn bei ihrer Verwendung in dieser Zeitschrift das Zeichen ${ }^{\circledR}$ oder ein anderer Hinweis auf etwa bestehende Schutz-rechte fehlen sollte. Für Satzfehler, insbesondere bei Dosierungs-angaben, wird keine Gewähr übernommen.

Die Zeitschrift sowie alle in ihr enthaltenen einzelnen Beiträge und Abbildungen sind urheberrechtlich geschützt. Jede Verwer-tung, die nicht ausdrücklich vom Urheberrechtsgesetz 
zugelassen ist, bedarf der vorherigen Zustimmung des Verlags. Das gilt insbesondere für Vervielfältigungen, Bearbeitungen, Übersetzun-gen, Mikroverfilmungen und die Einspeicherung und Verarbei-tung in elektronischen Systemen.

(C) Copyright 1987 by S. Karger Verlag für Medizin und Naturwissenschaften GmbH, Postfach 1724. D-8034 Germering

Satz und Druck: Walter Biering GmbH, Grafischer Betrieb, D-8000 München 45

Offizielles Organ

der Deutschen Gesellschaft für Hämatologie und Onkologie

und der

österreichischen Gesellschaft für Hämatologie und Onkologie

Offizielles Organ

der Österreichischen Krebsgesellschaft - Krebsliga 